\title{
Estimation of wind intensity data from reanalysis data using a shallow neural network
}

\author{
Dionisio Rodríguez-Esparragón \\ Instituto de Oceanografía y Cambio Global \\ Unidad Asociada ULPGC-CSIC \\ Las Palmas de Gran Canaria, Spain \\ dionisio.rodriguez@ulpg.es
}

\begin{abstract}
Global change is one of the outstanding problems nowadays. This is the reason why considerable attention, and economic resources to monitor climate variables have increased. Wind data constitute one of the key elements that determine the local climate. In this paper, the performance of a shallow neural net $(\mathrm{SNN})$ is tested to simulate remote sensing wind intensity data from reanalysis data from nearby location. As a result, a sequence of wind data with more spatial resolution can be achieved, allowing the availability of more data at the local scale.
\end{abstract}

Index Terms-shallow neural net, SNN, prediction, wind intensity, global change

\section{INTRODUCTION}

Climate change may be one of the most important global problems in the near decades [1]. Many atmospheric variables conform the climate of a region. Among them, winds play a crucial role. Specifically, the wind intensities, and direction condition the local weather and climate in oceanic regions [2]. This is the case of Canary Islands. The Canary's archipelago is placed in the Macaronesia region, near the west African coast, parallel to the Sahara Desert. Under these conditions, the predominant north-east Trade wind determines the climate of the islands [3].

In this context, analysing, monitoring, and estimating winds are important instruments to calibrate the evolution of global warming, and to predict and mitigate the problems associated to this phenomenon [4]-[6]. This issue is particularly important in local and small regions as those above described.

Aiming to analysing winds, scientific community can use long-term data from several reanalyses. However, reanalyses data is limited by the grid area, and local uncertainty is not resolved. Alternatively to reanalyses, data in situ offers a local approach to wind analysis and ad hoc studies. Nevertheless,

Funded by the Department of Economy, Industry, Commerce and Knowledge of the Canary Islands Government, and the European Fondo Europeo De Desarrollo Regional (FEDER).

\author{
Javier Marcello \\ Instituto de Oceanografía y Cambio Global \\ Unidad Asociada ULPGC-CSIC \\ Las Palmas de Gran Canaria, Spain \\ javier.marcello@ulpgc.es
}

\author{
Consuelo Gonzalo-Martín \\ Center for Biomedical Technology (CTB) \\ Universidad Politécnica de Madrid (UPM) \\ Madrid, Spain \\ consuelo.gonzalo@ctb.upm.es
}

local stations are not always available, and they need maintenance with associated costs. Also, specific remote sensing sensors have shown their capability to capture atmospheric data. In this case, the main drawbacks are the reduced life of satellite missions, and the relatively short history of this technology.

This work focuses in wind speed output provided by The NOAA ESRL Physical Sciences Division (PSD), and by The Physical Oceanography Distributed Active Archive Centre (PODAAC). The first one distributes a dataset of NCEP/NCAR reanalysis monthly means of this variable. The temporal range covers from 1948 to the present time with $2,5^{\circ} \times 2,5^{\circ}$ of spatial resolution. The second one generates a monthly mean ocean surface wind value to approximate a satellite-only climatological data record. The temporal range covers from 1987 to 2011 with $0,5^{\circ} \times 0,5^{\circ}$ of spatial resolution.

In this paper, a neural net is proposed to synthesize the PODAAC data using NCEP data as input. Thereby, wind data with higher spatial resolution that simulate real data can be derived. In this sense, the paper main contribution is the design and testing of this approach, and, additionally the contrast with other numerical perspectives.

The rest of this work is organized as follows: In section 2, the data used, and the applied methodology are introduced. In section 3, results are detailed and commented; finally, conclusion are established in section 4.

\section{DATA AND METHODOLOGY}

\section{A. Data}

Two points were selected for analysing in this study (NCEP, and PODAAC). They were chosen to satisfy two conditions: both points should be in oceanic waters, and the distance between them should be the shortest. Table 1 shows the latitude and longitude of the selected emplacements which are represented in Fig. 1. Both are located in the northwest of the Canary Islands, where we are conducting a deep study 
about winds evolution, and higher spatial resolution data were required.

TABLE I

GEOGRAPHICAL COORDINATES OF NCEP AND PODAAC SELECTED POINTS

\begin{tabular}{|c|c|c|}
\hline \multirow{2}{*}{ Source Data } & \multicolumn{2}{|c|}{ Geographical coordinates } \\
\cline { 2 - 3 } & Longitude & Latitude \\
\hline PODAAC & $-18.75^{\circ}$ & $29.5234^{\circ}$ \\
\hline NCEP & $-18.875^{\circ}$ & $29.625^{\circ}$ \\
\hline
\end{tabular}

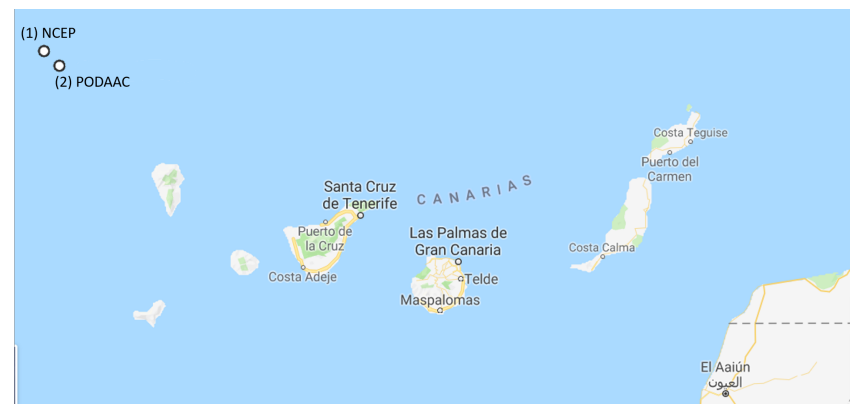

Fig. 1. Location of the analysed points relative to the Canary Islands.

The available data of these two points was downloaded from their respective official sources. In Fig. 2, the matching time interval between these NCEP and PODAAC data are represented as well as the absolute difference of the two sequences. Both time sequences cover 24 years of monthly average data, this means 2 sequences of 288 samples. The sequences have a calculated correlation coefficient of 0,922977933 , and the Root Mean Square Error (RMSE) is 0,847615428.

\section{B. Methodology}

The objective of this work includes answering the question about synthetizing PODAAC data using as reference NCEP data via a neural network approach. To this effect, the performance of this procedure must be contrasted against other numerical methods that can be applied. For these purposes, several techniques were executed: A first, analysis of the data was conducted to obtain the regression of NCEP versus PODAAC data using numerical techniques. Specifically, Linear, Tree, Support Vector machine and Gaussian Regression algorithms were applied [7]-[12]. Later, a shallow neural network (SNN) was designed to fit PODAAC data as response to NCEP input data.

SNN has extensively been applied to many fields. Forecasting seems to be one of the most extended application areas, ranging from economy science to electric systems, and including climatology [13]-[18]. Today, many research works are focused in analysing the performance of deep neural network (DNN) against SNN, but, at the moment, there is not consensus about which architecture outfit better this problem. SNN provide some interesting features for computing. They are fast and simple to program.

A SNN usually is composed of two layers (Fig. 3): hidden and output layers. Several tests were conducted to select the size of the hidden layer. As a result, 10 hidden neurons were chosen because this configuration generated the best fitting results between NCEP and PODAAC sequences. An input neuron and an output neuron complete the model. Training was executed with the LevenbergMarquardt algorithm (LMA) [19]. Although other algorithms were also tested (Bayesian regularization [20] and, conjugate gradient method [21]), LMA was chosen because of its better results. Finally, this design process was conducted by using $202(70 \%)$ of the samples to train the network, $43(15 \%)$ to validate the network, and, 43 $(15 \%)$ for testing purposes.

\section{RESUlTS}

The evaluation of the tested fitting algorithms appears in the table II. In this case, RMSE is calculated as the average squared difference between output of the SNN and original PODAAC data. While regression $\mathrm{R}$ values assess the correlation between the two cited variables. From table II, RMSE and $\mathrm{R}$ scores for SNN are the lowest and the closest to 1 respectively. This means that SNN performance to solve this problem seems to be the proper approach.

TABLE II

RESULTS OF THE EVALUATION OF REGRESSION ALGORITHMS BY COMPUTING RMSE AND R VALUES

\begin{tabular}{|c|c|c|c|}
\hline Evaluation & \multicolumn{3}{|c|}{ Regression Technique } \\
\cline { 2 - 4 } Technique & Linear & Tree & SVM \\
\hline RMSE & 0.74236 & 0.73524 & 0.70822 \\
\hline R & 0.85 & 0.86 & 0.87 \\
\hline & Ensemble & Gaussian & SNN \\
\hline RMSE & 0.72851 & 0.70428 & 0.63588 \\
\hline R & 0.86 & 0.87 & 0.92 \\
\hline
\end{tabular}

The $\mathrm{R}$ values for the training, validation and test data are shown in Fig. 4. Also, the fit between target and outputs for the different data subsets. All of them are over 0.9, which means that the designed SNN achieves a strong positive correlation.

Fig. 5.(a) shows the MSE resulting in a completed iteration of the training procedure (epoch). The MSE (RMSE) achieved were 0.3853 (0.6207), 0.4043 (0.6358) and 0.3037 (0.5511) respectively for the training, validation, and test data subsets. The histogram of the error is represented in Fig. 5.(b). Most of the errors are distributed around an interval of length inferior to 1 (from -0.4558 to 0.5064 ). This represents a $10.88 \%$ of the average of wind intensity in the NCEP sequence $(8.84 \mathrm{~m} / \mathrm{s})$.

Al last, PODAAC data ranging from 2012 to 2017 were synthetized in order to test the performance of the SNN. These results are displayed in Fig. 6 in contrast with source NCEP data as well as the error between the two sequences. The RMSE between both sequences is 0,631721348, and their coefficient of correlations is 0,984705465 . These values are close to the original training sequences as appear in section II.

\section{Conclusions}

The problem of synthetizing spatial distributed wind data from different sources by a SNN is addressed in this paper. 


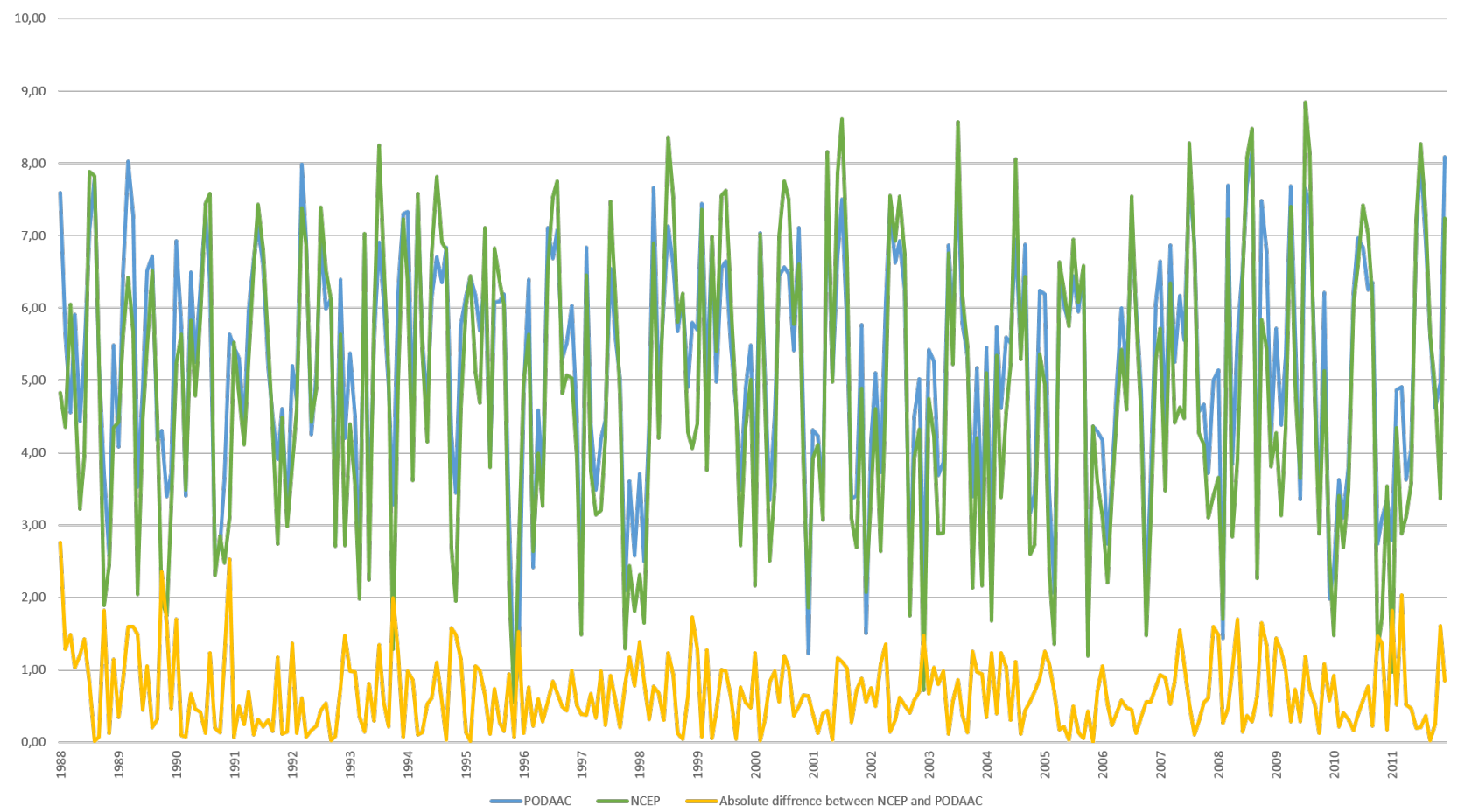

Fig. 2. Wind intensities (m/s) of NCEP (green) and PODAAC (blue) data from 1988 to 2011, and their difference (orange).

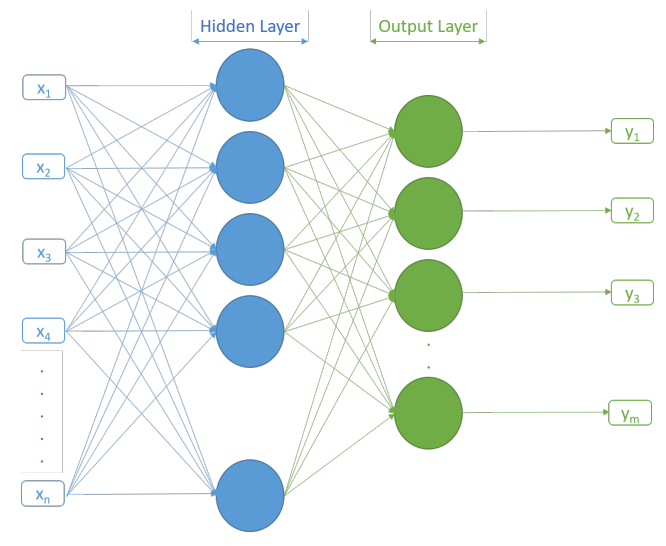

Fig. 3. Basic diagram of shallow neural network.

Specifically, we tried to find a prediction function to derive remote sensing satellite data (PODAAC) using as source reanalysis data (NCEP) which are located close geographically.

The SNN was trained with two original sequences of 24 years of wind intensity values. The performance of this approach was contrasted with other numerical algorithms. Specifically, computing of the RMSE, and the $\mathrm{R}$ values was conducted to assess the best prediction method. In this sense, SNN arises as the best way to tackle this problem as the qualitative results indicates. Moreover, a sequence of 5 years was obtained by executing the training neural net. Results match with the compared objective characteristics of original data.
The qualitative results shown in section 3 indicate that SNN can be considered a reliable solution in the context of this work. Moreover, their easy implementation and relatively low computational cost contribute to make an interesting approach to solve this prediction problem related to wind data. Further work is required to establish if this methodology can also be applied to wind direction data. Also, the contrast with a deep neural network approach should be addressed to analyse the performance improvement (if so) against simplicity of solution.

\section{ACKNOWLEDGMENT}

This work has been supported by the PASTOR (ProID2017010072) project, funded by the Department of Economy, Industry, Commerce and Knowledge of the Canary Islands Government, and the European Fondo Europeo de Desarrollo Regional (FEDER).

\section{REFERENCES}

[1] R. K. Pachauri, et al., Climate change 2014: synthesis report. Contribution of Working Groups I, II and III to the fifth assessment report of the Intergovernmental Panel on Climate Change. IPCC, 2014.

[2] R. D. Garreaud, and M. Falvey. "The coastal winds off western subtropical South America in future climate scenarios," International Journal of Climatology, vol. 29, no. 4, pp. 543-554, May 2008.

[3] F. Hernández, et al. "Influence of major African dust intrusions on the $137 \mathrm{Cs}$ and $40 \mathrm{~K}$ activities in the lower atmosphere at the Island of Tenerife," Atmospheric Environment, vol. 39, no 22, pp. 4111-4118, 2005.

[4] S. E. Belcher,J. N. Hacker, and D. S. Powell. "Constructing design weather data for future climates," Building services engineering research and technology, vol. 26, no 1, pp. 49-61, 2005.

[5] B. Van den Hurk, et al. "New climate change scenarios for the Netherlands," Water science and technology, vol. 56, no 4, pp. 27-33, 2007. 

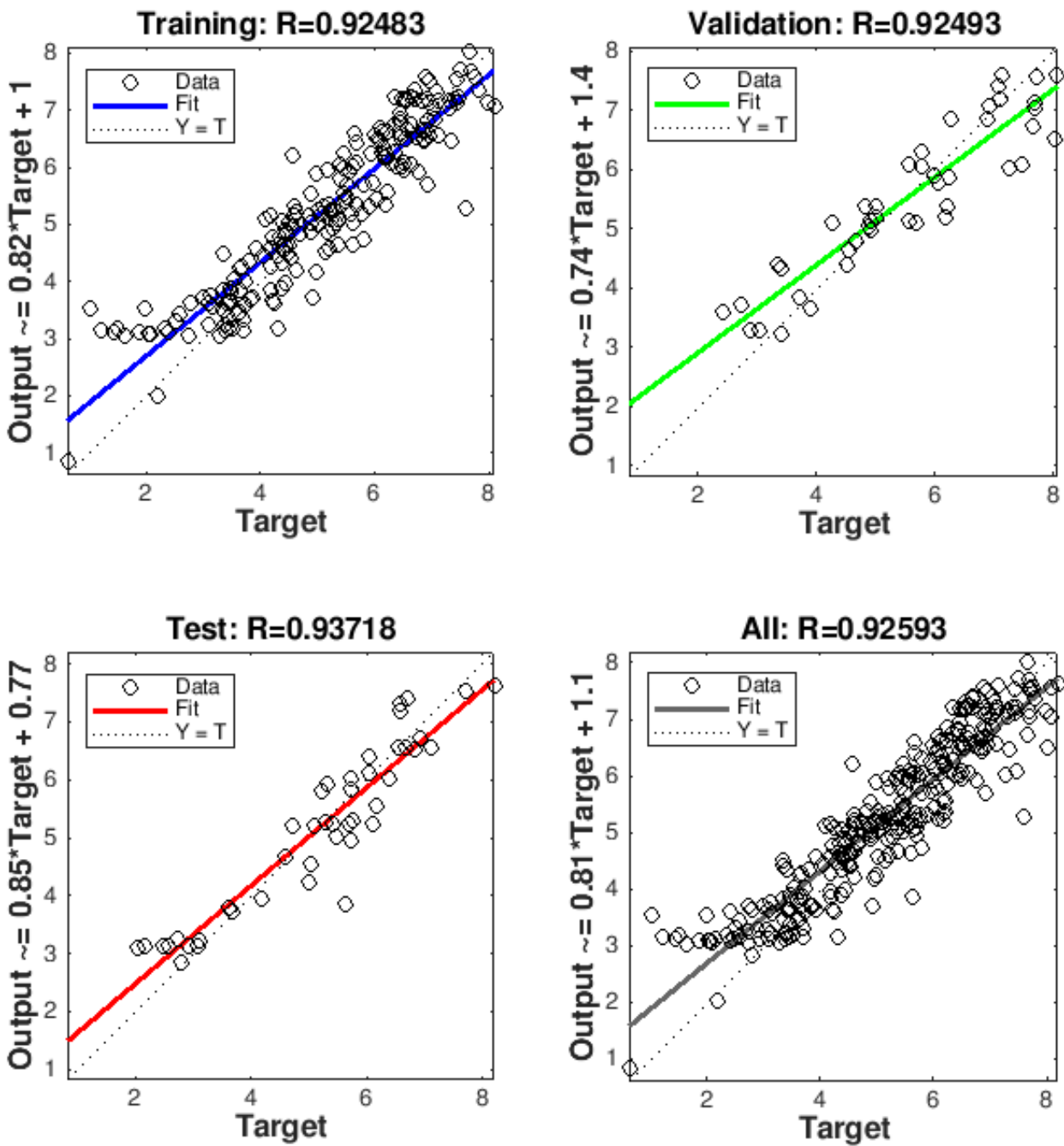

Fig. 4. Results of regression values for training, validation, and test data, as well as their average.

[6] S. C. Pryor, and R. J. Barthelemie, "Climate change impacts on wind energy: A review," Renewable and sustainable energy reviews, vol. 14, no 1, pp. 430-437, 2010.

[7] L. Breiman. "Bagging predictors," Machine learning, vol. 24, no 2, pp. $123-140,1996$

[8] J. Friedman, et al. "Additive logistic regression: a statistical view of boosting," The annals of statistics, vol. 28, no 2, pp. 337-407, 2000

[9] C. E. Rasmussen. "Gaussian processes in machine learning," In Summer School on Machine Learning. Springer, Berlin, Heidelberg, pp. 63-71, 2003

[10] A. J. Smola, and B. Scholkopf. "A tutorial on support vector regression," Statistics and computing, vol. 14, no 3, pp. 199-222, 2004.

[11] V. Kazemi, and J. Sullivan. "One millisecond face alignment with an ensemble of regression trees," Proceedings of the IEEE conference on computer vision and pattern recognition, pp. 1867-1874, 2014.

[12] L. Breiman.’Classification and regression trees," Routledge, 2017.

[13] C. D. Park, et al. "Electric load forecasting using an artificial neural network," IEEE transactions on Power Systems, vol. 6, no 2, pp. 442449, 1991

[14] I. Kaastra, and M. Boyd. "Designing a neural network for forecasting financial and economic time series," Neurocomputing, vol. 10, no 3, pp.
215-236, 1996.

[15] F. Tangang, W. Hsieh, and B. Tang. "Forecasting regional sea surface temperatures in the tropical Pacific by neural network models, with wind stress and sea level pressure as predictors," Journal of Geophysical Research: Oceans, vol. 103, no C4, pp. 7511-7522, 1988.

[16] T. Senjyu, et al. One-hour-ahead load forecasting using neural network," IEEE Transactions on power systems, vol. 17, no 1, pp. 113-118, 2002.

[17] G. P. Zhang. "Time series forecasting using a hybrid ARIMA and neural network model," Neurocomputing, vol. 50, pp. 159-175, 2003.

[18] G. P. Zhang, and M. QI. "Neural network forecasting for seasonal and trend time series," European journal of operational research, vol. 160, no 2, pp. 501-514, 2005.

[19] J, J, Moré. "The Levenberg-Marquardt algorithm: implementation and theory," Numerical analysis. Springer, Berlin, Heidelberg, pp. 105-116, 1978.

[20] F. D. Foresee, and M. T. Hagan. "Gauss-Newton approximation to Bayesian learning,". Proceedings of International Conference on Neural Networks (ICNN'97). IEEE, pp. 1930-1935, 1997.

[21] M. F. Moller. "A scaled conjugate gradient algorithm for fast supervised learning," Neural networks, vol. 6, no 4, pp. 525-533, 1993. 


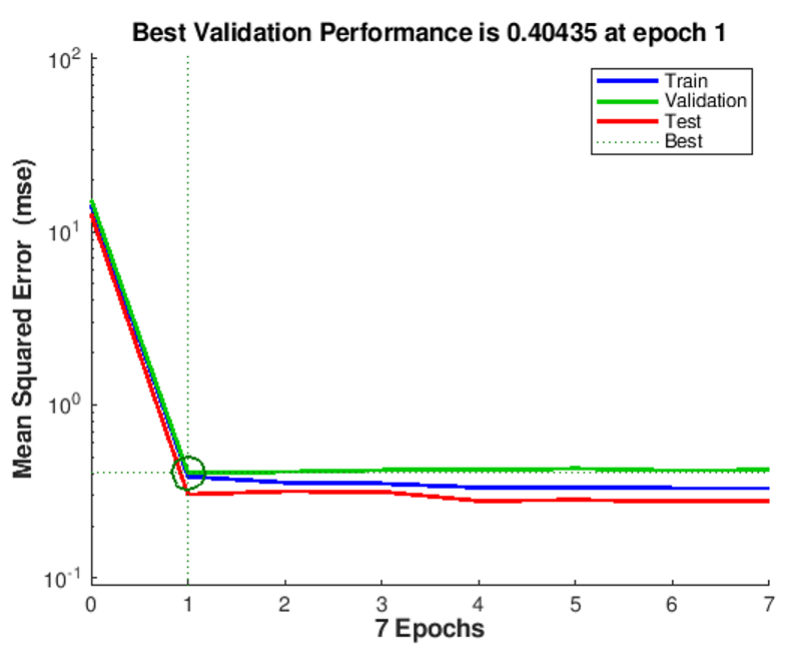

(a)

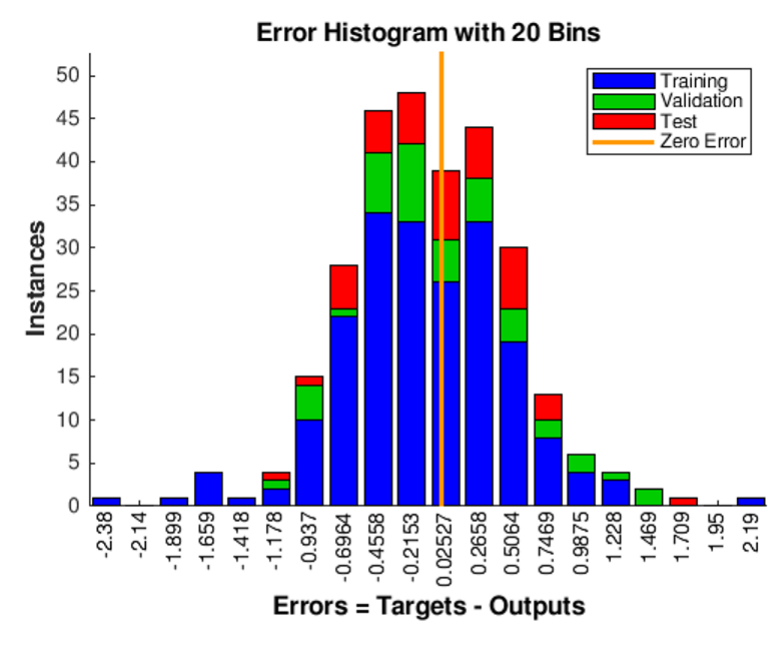

(b)

Fig. 5. Net perfomance: (a) MSE results versus training steps, (b) Distribution of the errors for training, validation, and test data.

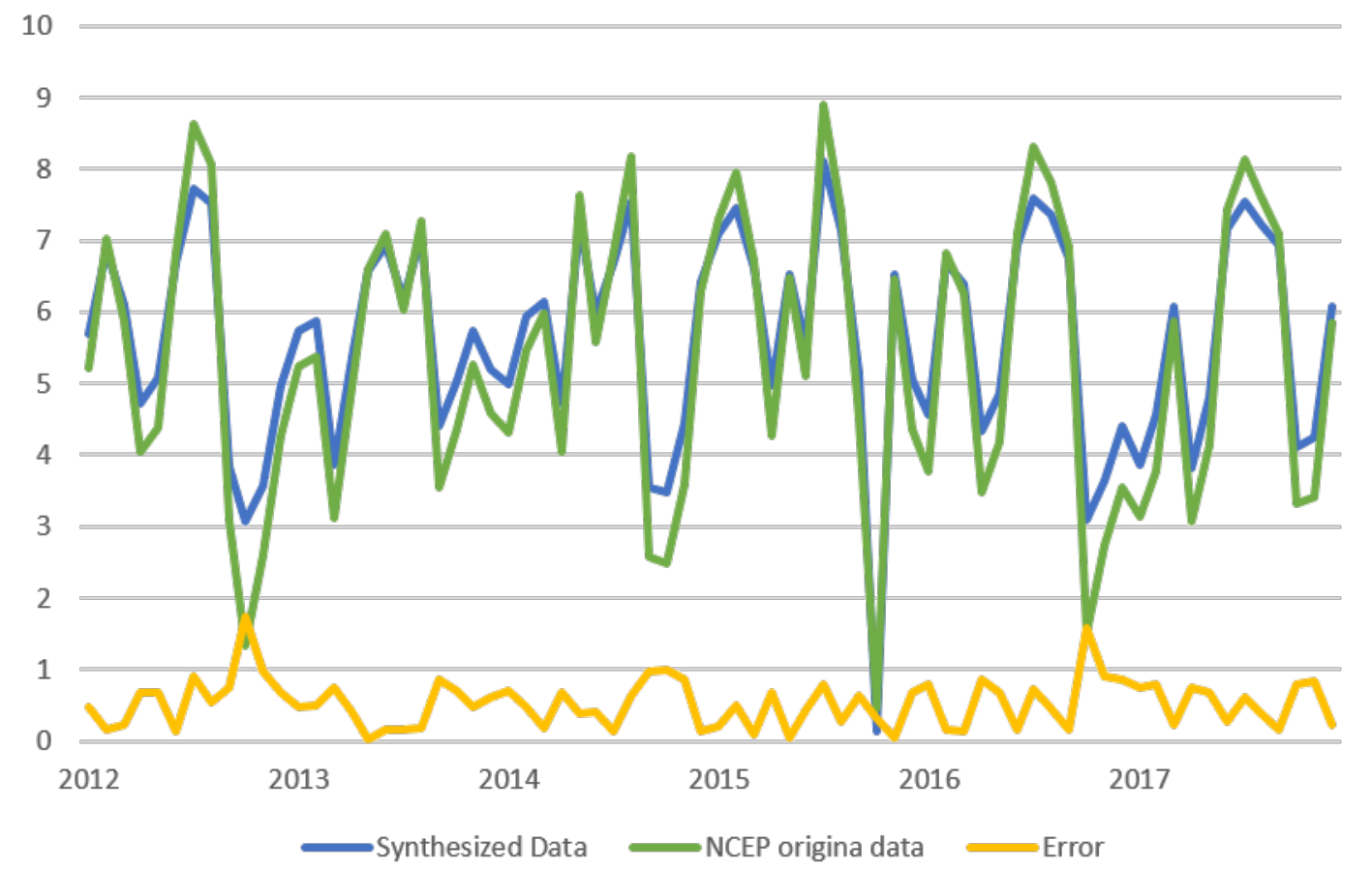

Fig. 6. Wind intensities (m/s) of NCEP (green) and synthetized (blue) data from 2012 to 2017, and their difference (orange). 
D.Rodríguez-Esparragón et al. • Estimation of Wind Intensity Data from Reanalysis Data using a Shallow Neural Network 